'Departamento de Psiquiatría, Escuela de Medicina, Facultad de Medicina, Universidad de Valparaíso, Valparaíso, Chile. 2Departamento de Psiquiatría, Escuela de Medicina, Facultad de Medicina, Universidad de Valparaíso, Valparaíso, Chile. ${ }^{3}$ Escuela de Medicina, Facultad de Medicina, Universidad de Valparaíso, Valparaíso, Chile. ${ }^{4}$ Escuela de Medicina, Facultad de Medicina, Universidad de Valparaíso, Valparaíso, Chile. ${ }^{5}$ Departamento de Salud Pública, Escuela de Medicina, Facultad de Medicina, Universidad de Valparaíso, Valparaíso, Chile.

${ }^{6}$ Centro de Investigaciones Biomédicas, Escuela de Medicina, Facultad de Medicina, Universidad de Valparaíso, Valparaíso, Chile.

Recibido el 23 de mayo de 2016, aceptado el 22 de octubre de

Correspondencia a: Eva Madrid Aris MD, PhD, Escuela de Medicina, Facultad de Medicina, Universidad de Valparaíso, Valparaíso, Chile. Angamos 655, Viña del Mar, Chile.

Teléfono: + 56322603099 eva.madrid@uv.cl

\section{Funcionamiento psicosocial en pacientes agudos y crónicos no psiquiátricos en régimen hospitalario: depresión, alexitimia y falta de asertividad}

\author{
MARCELO ARANCIBIA ${ }^{1}$, ROSA BEHAR ${ }^{2}$, SOFÍA MARÍN ${ }^{3}$, \\ NICOLÁS INZUNZA ${ }^{4}$, EVA MADRID ${ }^{5,6}$
}

Background: Depression, alexithymia, and lack of assertiveness interfere with individual psychosocial functioning and may result in longer hospitalization stay and poorer therapeutic results. Aim: To analyze the psychosocial functioning in acute and chronic patients and its association with psychological, clinical and sociodemographic variables. Material and Methods: We performed a cross-sectional study that included 80 inpatients of both sexes with organic pathology, aged between 18 to 70 years old, without any current psychiatric disorder. Clinical and sociodemographic data were collected from a semi-structured interview and hospital records. Beck Depression Inventory-IA, Toronto Alexithymia Scale-20 and Rathus Assertiveness Scale were administered. Results: Fifty five percent of patients had some degree of depression, 33\% alexithymia and 34\% lack of assertiveness. The levels of depression, alexithymia and lack of assertiveness in chronic patients were significantly higher than those observed in acute patients. Women and participants older than 60 years exhibited the highest degrees of depression. Alexithymia and lack of assertiveness were associated with a lower educational level. A negative significant correlation between alexithymia and assertiveness scores was observed among acute patients. Conclusions: Participants with chronic diseases had a lower psychosocial functioning. Less educated patients showed more alexithymic and less assertive features. We emphasized the need of a better management of these aspects by the health team, since social functioning might interfere with the outcome of physical illnesses.

(Rev Med Chile 2016; 144: 1424-1431)

Key words: Affective Symptoms; Assertiveness; Depression; Inpatients; Mood Disorders.

El funcionamiento psicosocial individual es un determinante central en el diagnóstico, evolución, tratamiento y pronóstico de diversas patologías orgánicas, pudiendo ser evaluado mediante la disfunción de algunos de sus componentes, tales como la depresión, la alexitimia y la falta de asertividad ${ }^{1}$.

Los trastornos depresivos conforman un pro- 
blema de salud pública debido a su alta prevalencia y a las repercusiones físicas, psicológicas, sociales y económicas que conllevan². Según la Organización Mundial de la Salud, la depresión es la principal causa de discapacidad y limitaciones funcionales, superando a patologías como la hipertensión arterial, la artritis reumatoide y la diabetes mellitus ${ }^{2-7}$. La sintomatología depresiva es extremadamente frecuente entre hospitalizados ${ }^{2}$, alcanzando cifras de prevalencia de hasta $50 \%$, en contraste al 5\% reportado por la población general ${ }^{3,5,8}$. Este fenómeno sería un predictor adecuado de pobres resultados terapéuticos, estadías hospitalarias más prolongadas y menor adherencia al tratamiento ${ }^{5,6}$.

La alexitimia, fenómeno frecuentemente confundido con la depresión, consiste en la dificultad para identificar y describir sentimientos y distinguirlos de las sensaciones corporales del arousal (activación) emocional, con una constricción del pensamiento simbólico y un estilo cognitivo orientado hacia el espacio externo y concreto ${ }^{9}$. Esta condición, descrita clásicamente en enfermos psicosomáticos, presenta actualmente un reconocido rol como desencadenante, perpetuante e índice pronóstico de ciertas enfermedades crónicas ${ }^{6,10,11}$, mediante una mayor susceptibilidad al estrés, el aumento de conductas poco saludables y la propensión a desórdenes inmunomediados ${ }^{11}$. A su vez, se ha propuesto una íntima relación entre este constructo y la falta de asertividad, asociada a rasgos de sumisión o timidez y sentimientos de ineficacia personal, manifestándose como una agresión auto-dirigida por medio de la culpa y/o crítica, incompetencia para expresar juicios discrepantes y hacer valer las emociones y sentimientos propios $^{12}$. Así, la depresión, la alexitimia y la falta de asertividad se asociarían por una parte a numerosas entidades nosológicas, como el cáncer, las enfermedades cardiovasculares y la diabetes mellitus, y por otra, con los costos y resultados hospitalarios ${ }^{8,10,11,13-15}$. Sin embargo, estas relaciones son aspectos insuficiente e inadecuadamente estudiados y/o tratados, ya que su investigación se ha enfocado mayoritariamente en población psiquiátrica y con menor frecuencia en muestras de enfermos somáticos en régimen hospitalario ${ }^{1}$.

En virtud de lo expuesto, se estudiará la frecuencia y severidad de depresión, alexitimia y falta de asertividad en pacientes agudos (PA) y crónicos (PC) internados en los Servicios de Medicina Interna, Oncología y Traumatología del Hospital
Carlos van Buren de Valparaíso, analizando su relación con variables clínicas y sociodemográficas.

\section{Material y Método}

\section{Diseño}

Se desarrolló un estudio de corte transversal para caracterizar comparativamente el perfil de funcionamiento psicosocial en PA y PC hospitalizados.

\section{Muestra}

La muestra quedó conformada por adultos hospitalizados durante abril y mayo de 2015 en los Servicios de Medicina Interna, Oncología y Traumatología del Hospital Carlos van Buren de Valparaíso, considerando a todos aquéllos que cumplieran los siguientes criterios de inclusión: 1) Pacientes hospitalizados por un período igual o superior a cinco días; 2) Hospitalizados por una patología orgánica; 3) Mayores de 18 años y 4) Con capacidad cognitiva conservada. Los criterios de exclusión abarcaron: 1) Pacientes que no aceptaron ser parte del estudio; 2) Que no fuesen capaces de responder los cuestionarios aplicados; 3) Mayores de 70 años; 4) Embarazadas y 5) Con diagnóstico actual de algún trastorno psiquiátrico.

Se consideró el cálculo del tamaño de la muestra según un alfa $\leq 0,05$ y un poder estadístico de 0,80 en función de los puntajes considerados para la población normal dados por la Escala de Alexitimia de Toronto-20, estimándose un mínimo de 28 individuos por grupo de comparación para demostrar una diferencia de $10 \%$ del valor de alexitimia.

\section{Procedimientos}

Previa autorización del estudio por parte del Comité Científico del Hospital Carlos van Buren y aceptación del consentimiento informado por los pacientes, fueron aplicados el Inventario de Depresión de Beck-IA (BDI-IA) ${ }^{16}$, la Escala de Alexitimia de Toronto-20 (TAS-20) ${ }^{17}$ y la Escala de Asertividad de Rathus (RAS) ${ }^{18}$ (Tabla 1).

Adicionalmente, se efectuaron entrevistas semi-estructuradas para la recopilación de datos demográficos y clínicos (edad, estado civil, escolaridad, ocupación, días de estadía hospitalaria, enfermedad médica actual, antecedentes de atención psicológica y/o psiquiátrica o de patología mental, 
Tabla 1. Descripción de instrumentos

Inventario de Depresión de Beck-IA (Beck Depression Inventory-IA) (BDI-IA) ${ }^{16}$

- 21 preguntas auto-aplicables con cuatro frases indicativas de graduación de severidad de síntomas depresivos, permitiendo clasificar el nivel de depresión en leve, intermitente, moderada, grave y extrema

- Cada respuesta se valora entre 0 y 3 puntos

- Los puntajes se corresponden según: 1-10 depresividad normal, 11-16 leve, 17-20 intermitente, 21-30 moderada, 31-40 grave $y \geq 41$ extrema

Escala de Alexitimia de Toronto (Toronto Alexithymia Scale-20) (TAS-20) ${ }^{17}$

- 20 preguntas auto-aplicables que evalúan el grado de alexitimia, distribuyéndose en 3 subescalas: T1) Dificultad en identificar sentimientos y en distinguir entre sentimientos y sensaciones corporales de la activación emocional; T2) Dificultad en la expresión verbal de las emociones y T3) Pensamiento orientado hacia detalles externos

- Cada respuesta posee valores entre 0 y 5 puntos

- Puntajes $>52$ y $<59$ indican alexitimia baja, y $\geq 59$ alexitimia alta

Escala de Asertividad de Rathus (Rathus Assertiveness Scale) (RAS) ${ }^{18}$

- 30 ítems auto-aplicables que evalúan el grado de asertividad en una variedad de situaciones y en términos de autoexpresión en tres factores: R1) Conductas deficitarias como la timidez, la ansiedad paralizante y todas las expresiones injustificadas de fracaso; R2) Sinceridad emocional, dominación efectiva con el otro y confrontación positiva con eventos sociales y R3) Conductas de sumisión y dependencia

- Escala de puntaje de +3 a -3 , excluyendo 0

- Puntajes entre -90 (extremadamente no asertivo) hasta +90 (extremadamente asertivo)

uso de psicofármacos y consumo de sustancias). Los antecedentes fueron corroborados a partir de la revisión de fichas clínicas.

\section{Análisis de datos}

Se utilizaron medias, desviaciones estándar y proporciones para el análisis descriptivo. Para el análisis inferencial se compararon medias en dos grupos a través de la prueba $t$ de Student, mientras que para la comparación de variables categóricas se aplicó la prueba exacta de Fisher. Para contrastar dos variables cuantitativas se usó el índice r de correlación de Pearson. Los datos se analizaron usando el software computacional Stata 12 (Statacorp USA).

\section{Resultados}

Se incluyeron 80 participantes de entre 19 y 70 años (51,3 años promedio), siendo categorizados en dos grupos según el carácter de la instalación temporal del motivo de hospitalización actual, definiéndose un grupo de PA y otro de PC. No hubo diferencias significativas intergrupales entre las variables estado civil, escolaridad, ocupación, días de estadía hospitalaria, antecedentes psiquiátricos individuales y consumo de sustancias (Tabla 2). Se encontraron diferencias de las características basales de los dos grupos en las variables edad, número de hospitalizaciones previas y consumo de alcohol: los PC presentaron edad y número de hospitalizaciones previas significativamente mayores que los $\mathrm{PA}$, y estos últimos reportaron ser consumidores de alcohol en una proporción dos veces mayor que la de los PC.

El puntaje promedio de BDI-IA del total de la muestra fue de 13,4 (DE $\pm 8,4)$, exhibiendo un $55 \%$ algún grado de depresión. Una proporción significativamente más elevada de pacientes mayores de sesenta años mostró algún nivel de depresión en relación a aquéllos menores $(68,8 \%$ versus $45,8 \%$ - $p=0,036$ ). Asimismo, el porcentaje de mujeres que reportó algún nivel de depresión fue significativamente superior que la de hombres $(66,7 \%$ versus $42,1 \%-p=0,024)$, presentando puntajes promedio también superiores ( 15 versus $11,6-p=0,031)$. Por otra parte, el puntaje promedio de TAS-20 de la muestra total fue de 41,1 (DE $\pm 21,5)$, portando 32,5\% algún nivel de alexitimia. Se hallaron diferencias significativas $(\mathrm{p}<0,001)$ en los puntajes promedios de TAS-20 entre aquéllos con escolaridad básica/media $(47,8$ puntos - $\mathrm{DE} \pm 19,8)$ y quienes contaban con edu- 
Tabla 2. Antecedentes sociodemográficos y clínicos de PA y PC

\begin{tabular}{|c|c|c|c|c|c|}
\hline & \multicolumn{2}{|c|}{$\begin{array}{c}\text { PA } \\
(N=33)\end{array}$} & \multicolumn{2}{|c|}{$\begin{array}{c}P C \\
(N=47)\end{array}$} & Valor $p$ \\
\hline Edad & \multicolumn{2}{|c|}{$47,3( \pm 15,9)$} & \multicolumn{2}{|c|}{$54,9( \pm 14,6)$} & 0,002 \\
\hline Sexo (Femenino) & \multicolumn{2}{|c|}{$17(51,5 \%)$} & \multicolumn{2}{|c|}{$25(53,2 \%)$} & NS \\
\hline $\begin{array}{l}\text { Estado civil (casados/ } \\
\text { convivientes) }\end{array}$ & \multicolumn{2}{|c|}{$17(51,5 \%)$} & \multicolumn{2}{|c|}{$26(55,3 \%)$} & NS \\
\hline Escolaridad & $\begin{array}{l}\text { Básica } \\
\text { Media } \\
\text { Técnico-universitaria }\end{array}$ & $\begin{array}{c}3(9 \%) \\
18(54,5 \%) \\
12(36,3 \%)\end{array}$ & $\begin{array}{l}\text { Básica } \\
\text { Media } \\
\text { Técnico-universitaria }\end{array}$ & $\begin{array}{r}15(31,9 \%) \\
23(48,9 \%) \\
9(19,1 \%)\end{array}$ & NS \\
\hline Ocupación & $\begin{array}{l}\text { Trabajando actualment } \\
\text { Jubilados } \\
\text { Dueñas de casa } \\
\text { Estudiantes }\end{array}$ & $\begin{array}{r}21(63,6 \%) \\
3(9,1 \%) \\
5(15,2 \%) \\
4(12,1 \%)\end{array}$ & $\begin{array}{l}\text { Trabajando actualme } \\
\text { Jubilados } \\
\text { Dueñas de casa } \\
\text { Estudiantes }\end{array}$ & $\begin{array}{cl}22 & (46,8 \%) \\
8 & (17 \%) \\
13 & (27 \%) \\
4 & (8,5 \%)\end{array}$ & NS \\
\hline Días de estadía & \multicolumn{2}{|c|}{10,2 días $( \pm 15,2)$} & \multicolumn{2}{|c|}{11,4 días $( \pm 13,5)$} & NS \\
\hline $\begin{array}{l}\text { Antecedentes personales } \\
\text { de atención psicológica }\end{array}$ & \multicolumn{2}{|c|}{$10(30,3 \%)$} & \multicolumn{2}{|c|}{$12(25,5 \%)$} & NS \\
\hline $\begin{array}{l}\text { Antecedentes personales } \\
\text { de atención psiquiátrica }\end{array}$ & \multicolumn{2}{|c|}{$4(12,1 \%)$} & \multicolumn{2}{|c|}{$6(12,8 \%)$} & NS \\
\hline Uso de psicofármacos & \multicolumn{2}{|c|}{$4(12,1 \%)$} & \multicolumn{2}{|c|}{$3(6,4 \%)$} & NS \\
\hline Tabaquismo & \multicolumn{2}{|c|}{$12(36,4 \%)$} & \multicolumn{2}{|c|}{$9(19,2 \%)$} & NS \\
\hline Consumo de alcohol & \multicolumn{2}{|c|}{$19(57,6 \%)$} & \multicolumn{2}{|c|}{$13(27,7 \%)$} & 0,007 \\
\hline Consumo de cannabis & \multicolumn{2}{|c|}{$7(21,2 \%)$} & \multicolumn{2}{|c|}{$6(12,8 \%)$} & NS \\
\hline $\begin{array}{l}\text { Número de hospitalizaciones } \\
\text { previas }\end{array}$ & \multicolumn{2}{|c|}{$1,36( \pm 1,8)$} & \multicolumn{2}{|c|}{$3,47( \pm 3,46)$} & 0,002 \\
\hline
\end{tabular}

NS = no significativo. Valores expresados en promedio y proporciones (DE). Comparación usando prueba t Student y prueba exacta de Fisher.

cación técnica/universitaria (22,2 puntos - DE \pm 13,1). Para la totalidad de la muestra, se obtuvo un RAS total de $12,9(\mathrm{DE} \pm 32,2)$, calificando como no asertivos $33,8 \%$ de los sujetos. El RAS total del grupo con educación básica/media fue significativamente menor que el demostrado por quienes presentaban educación técnica/universitaria $(8$ versus 26,5 puntos $-\mathrm{p}=0,02$ ). No se encontró significación estadística entre los puntajes totales o factoriales de BDI-IA, TAS-20 y RAS y el resto de las variables sociodemográficas evaluadas. Los puntajes para BDI-IA, TAS-20 y RAS según PA y PC se señalan en Tabla 3.

El índice de correlación de Pearson demostró correlaciones negativas significativas para PA entre los puntajes totales de TAS-20 y RAS $(r=-0,64)$, TAS-20 y R2 $(r=-0,63)$ y T2 y R2 $(r=-0,67)$ (Tablas 4 y 5 ).

\section{Discusión}

Nuestros hallazgos destacan que los PC exhiben mayores niveles de depresión, alexitimia y falta de asertividad que los PA. Asimismo, se encontraron grados aumentados de depresión entre la población hospitalizada femenina y mayor de sesenta años, y tanto la alexitimia como la falta de asertividad fueron más altas a menor grado de escolaridad. Paralelamente, hubo correlaciones significativas en PA entre los puntajes de TAS-20 y RAS, TAS -20 y R2 y T2 y R2.

Un 55\% de la muestra presentó algún nivel de depresión, informando un $21 \%$ características compatibles con depresión moderada y $5 \%$ grave, superior al $14 \%$ descrito por Katon ${ }^{19}$, pero similar al $50 \%$ señalado por Campo y cols. ${ }^{8}$ en población hospitalaria. Según la formulación ori- 
Tabla 3. Depresión, alexitimia y asertividad en PA y PC

\begin{tabular}{|c|c|c|c|}
\hline & $\begin{array}{c}\text { PA } \\
(\mathbf{N}=\mathbf{3 3})\end{array}$ & $\begin{array}{c}P C \\
(N=47)\end{array}$ & Valor p \\
\hline Puntaje total BDI-A & $10,4 \quad( \pm 7,3)$ & $15,5 \quad( \pm 8,5)$ & 0,006 \\
\hline Presencia de algún grado de depresión & $42,4 \%$ & $63,8 \%$ & 0,048 \\
\hline Puntaje total TAS-20 & $30,3( \pm 16,8)$ & $48,7 \quad( \pm 21,2)$ & $<0,001$ \\
\hline Presencia de algún grado de alexitimia & $12 \%$ & $46,8 \%$ & 0,001 \\
\hline Puntaje T1 & $6,6 \quad( \pm 6,6)$ & $14,6 \quad( \pm 10,6)$ & $<0,001$ \\
\hline Puntaje T2 & $7,7 \quad( \pm 7,8)$ & $13,1 \quad( \pm 7,1)$ & 0,002 \\
\hline Puntaje T3 & $15,9 \quad( \pm 7,4)$ & $20,8 \quad( \pm 7,9)$ & 0,007 \\
\hline Puntaje total RAS & $30,2( \pm 30,7)$ & $0,72( \pm 27,7)$ & $<0,001$ \\
\hline Presencia de falta de asertividad & $18,2 \%$ & $44,7 \%$ & 0,012 \\
\hline Puntaje R1 & $8,6 \quad( \pm 7,3)$ & $0,6 \quad( \pm 9,1)$ & $<0,001$ \\
\hline Puntaje R2 & $10,7 \quad( \pm 8,8)$ & $1,8 \quad( \pm 9,3)$ & $<0,001$ \\
\hline Puntaje R3 & $6,6( \pm 11,4)$ & $-2,9 \quad( \pm 8,9)$ & $<0,001$ \\
\hline
\end{tabular}

Valores expresados en promedio y proporciones (DE). Comparación usando prueba t Student y prueba exacta de Fisher. Inventario de Depresión de Beck I-A (BDI-IA). Escala de Alexitimia de Toronto-20 (TAS-20): T1: Dificultad en identificar sentimientos y en distinguirlos de la activación emocional; T2: Dificultad en la expresión verbal emocional; T3: Pensamiento orientado hacia detalles externos. Escala de Asertividad de Rathus (RAS): R1: Timidez y ansiedad paralizante; R2: Sinceridad emocional y confrontación positiva con eventos sociales; R3: Sumisión y dependencia.

\section{Tabla 4. Valores de correlación entre puntajes de BDI-IA, TAS-20 y RAS en PA (expresados en índice $r$ de correlación de Pearson)}

\begin{tabular}{|lccccc|}
\hline & BDI-IA & TAS-20 & T1 & T2 & T3 \\
TAS & 0,28 & & & & \\
T1 & 0,04 & & & & \\
T2 & 0,28 & & & & \\
T3 & 0,29 & & & & \\
RAS & $-0,17$ & $-0,64$ & $-0,44$ & $-0,54$ & $-0,48$ \\
R1 & $-0,31$ & $-0,44$ & $-0,27$ & $-0,32$ & $-0,40$ \\
R2 & $-0,28$ & $-0,63$ & $-0,41$ & $-0,67$ & $-0,35$ \\
R3 & 0,11 & $-0,20$ & $-0,26$ & $-0,09$ & $-0,12$ \\
\hline
\end{tabular}

Inventario de Depresión de Beck I-A (BDI-IA). Escala de Alexitimia de Toronto-20 (TAS-20): T1: Dificultad en identificar sentimientos y en distinguirlos de la activación emocional; T2: Dificultad en la expresión verbal emocional; T3: Pensamiento orientado hacia detalles externos. Escala de Asertividad de Rathus (RAS): R1: Timidez y ansiedad paralizante; R2: Sinceridad emocional y confrontación positiva con eventos sociales; R3: Sumisión y dependencia.
Tabla 5. Valores de correlación entre puntajes de BDI-IA, TAS-20 y RAS en PC (expresados en índice $r$ de correlación de Pearson)

\begin{tabular}{|lccccc|}
\hline & BDI-IA & TAS-20 & T1 & T2 & T3 \\
TAS & 0,47 & & & & \\
T1 & 0,48 & & & & \\
T2 & 0,38 & & & & \\
T3 & 0,25 & & & & \\
RAS & $-0,27$ & $-0,50$ & $-0,26$ & $-0,45$ & $-0,58$ \\
R1 & $-0,34$ & $-0,57$ & $-0,46$ & $-0,43$ & $-0,50$ \\
R2 & $-0,25$ & $-0,48$ & $-0,19$ & $-0,49$ & $-0,58$ \\
R3 & $-0,24$ & $-0,29$ & $-0,11$ & $-0,30$ & $-0,33$ \\
\hline
\end{tabular}

Inventario de Depresión de Beck I-A (BDI-IA). Escala de Alexitimia de Toronto-20 (TAS-20): T1: Dificultad en identificar sentimientos y en distinguirlos de la activación emocional; T2: Dificultad en la expresión verbal emocional; T3: Pensamiento orientado hacia detalles externos. Escala de Asertividad de Rathus (RAS): R1: Timidez y ansiedad paralizante; R2: Sinceridad emocional y confrontación positiva con eventos sociales; R3: Sumisión y dependencia. 
ginal del BDI-IA ${ }^{20}$, ya los portadores de depresión moderada deberían recibir atención profesional, hallados sobre todo en el grupo de PC. Este hecho destacala necesidad de la existencia de unidades de psiquiatría de enlace, pues la enfermedad crónica es un factor de riesgo permanente para depresión, independientemente de la fisiopatología subyacente $^{21}$. Una revisión sistemática reveló que los síntomas depresivos en pacientes hospitalizados fueron indicadores de hospitalizaciones subsecuentes $^{21}$, retroalimentándose un círculo que tiene por una parte a la enfermedad crónica, que con lleva per se hospitalizaciones múltiples, y por otra, al trastorno depresivo, mantenedor de un estado de salud deficitario. La depresión en un contexto médico-quirúrgico suele sub-diagnosticarse, dada la confusión generada a partir de la sintomatología intercurrente explicada por la patología de base ${ }^{14}$, efectos farmacológicos secundarios y desconocimiento del trastorno en el personal sanitario ${ }^{22,23}$, el que interpretaría los síntomas depresivos como congruentes con el estrés hospitalario ${ }^{24}$ y tendería a reconocer la depresión sólo a través de su vertiente afectiva, en desmedro de la cognitiva, conductual y psicomotora ${ }^{5}$. Asimismo, adultos mayores y mujeres presentarían una serie de atributos psicológicos similares al estado depresivo, y culturalmente considerados como inherentes a estos grupos, imposibilitándose una pesquisa apropiada. En la exploración presente, mujeres y mayores de 60 años exhibieron niveles más altos de depresión, conjuntos que paralelamente presentan frecuencias mayores de enfermedades crónicas ${ }^{25}$.

El estado actual de la alexitimia es ampliamente discutido, pues se ha señalado como rasgo estable independiente de la depresión y como fenómeno de estado secundario a ella9 ${ }^{9}$. En este sentido, si bien se ha puntualizado que el TAS-20 mediría más bien afectos depresivos como angustia, culpa $y$ tristeza ${ }^{26}$, en este trabajo no se observaron correlaciones altas entre las puntuaciones de BDI-IA y TAS-20. Dentro de los PA, 12\% fueron alexitímicos, en tanto que $46,8 \%$ de los PC cumplieron con la condición, con valores significativamente mayores en los tres factores de TAS-20. Efectivamente, $60 \%$ de los enfermos somáticos crónicos presentaría manifestaciones psicopatológicas asociadas a la depresión, el bloqueo emocional y el perfil alexitímico ${ }^{27}$. Particularmente, los PC alexitímicos evidenciarían un riesgo de mortalidad dos veces mayor que los no alexitímicos, aumentando $1,2 \%$ por cada punto del TAS-20 en enfermedades cardiovasculares $^{11,28}$.

Algunos estudios apoyan una superposición entre variables sociodemográficas y alexitimia ${ }^{29,30}$, y otros la vinculan sólo a un número limitado de factores sociales ${ }^{31}$. Honkalampi et a ${ }^{32}$ verificaron que $10 \%$ de la población general sería alexitímica, con una frecuencia significativamente superior entre los hombres, diferencia no pesquisada en esta investigación. Adicionalmente, los autores hallaron que los alexitímicos tuvieron una significativa menor escolarización, aseveración concordante con nuestro registro, donde la categoría educacional básico-media exhibió cifras de alexitimia mayores al doble de las expuestas por el grupo técnico-universitario, hecho confirmado por investigadores japoneses, quienes detectaron asociaciones significativas inversas entre alexitimia y nivel educacional y socioeconómico ${ }^{33}$. Así, las variables sociales explicarían 9\% de la varianza en los puntajes del TAS- $20^{29}$, pues la limitación económica impactaría en una mayor privación sociocultural instructiva, constituyendo una vulnerabilidad para el desarrollo de rasgos alexitímicos en relación a la menor adquisición de un vocabulario complejo para la notificación emocional $^{27}$. En nuestro análisis, esto se reflejaría en que el pensamiento concreto (T3) fue el factor del TAS-20 que mayor puntaje alcanzó tanto en PA como en PC.

La dificultad en el reconocimiento afectivo podría reducir notoriamente la expresividad emocional $^{12}$, característica central de la falta de asertividad, cuya frecuencia en PC $(44,7 \%)$ fue significativamente mayor que en PA $(18,2 \%)$, al igual que lo sucedido con la alexitimia, siendo en ambos tipos de pacientes la sumisión y la dependencia los rasgos preponderantes, significativamente mayores en PC $(p<0,001)$ y con menor escolaridad. No obstante, la relación entre alexitimia y déficit de auto-aserción fue más prominente entre PA, pues se constataron correlaciones negativas altas entre los puntajes totales de alexitimia y nivel de asertividad $(r=-0,64)$, de alexitimia y sinceridad emocional $(r=-0,63)$ y de esta última con dificultad en la expresión verbal de las emociones $(\mathrm{r}=$ $-0,67)$, lo que contribuiría con una mala gestión de estados emocionales, falta de reconocimiento y notificación de síntomas somáticos, y de esta manera con peor pronóstico evolutivo. 


\section{Limitaciones, Implicancias y Conclusiones}

El contexto hospitalario presenta fenómenos agregados que pueden haber afectado la correcta notificación de los pacientes, generando consecuentemente informes menos apropiados. Debe considerarse que la evaluación de la alexitimia por medio de cuestionarios de auto-reporte estaría teóricamente limitada, pues por definición los alexitímicos no deberían ser capaces de informar sus estados psicológicos adecuadamente. Además, la falta de evidencia disponible sobre los parámetros analizados dificultó un mejor análisis de los datos obtenidos desde un enfoque comprensivo.

Se presenta por primera vez el estudio de parámetros como alexitimia y asertividad en población hospitalaria en Chile, contribuyendo con la escasa suma de exploraciones sobre el funcionamiento psicosocial de pacientes internados. Se ha corroborado la alta prevalencia de estas características en PC, quienes constituyen una alta proporción del volumen hospitalario, pesquisándose fenómenos directamente relacionados con peores resultados terapéuticos y mayores costos sanitarios. Se hace necesario un mayor conocimiento y reconocimiento de estos aspectos por parte del equipo de salud, paralelamente al establecimiento de unidades de psiquiatría de enlace en hospitales generales, para dar respuesta a esta dimensión, muchas veces relegada tras el cuidado de la esfera somática del enfermo. Esta contingencia afectaría paradójicamente la evolución y el desenlace de la dolencia física. El presente estudio enfatiza la comprensión del paciente como un todo, con consciencia de la interacción bidireccional mente-cuerpo, enfoque especialmente efectivo en el tratamiento de enfermedades crónicas.

\section{Referencias}

1. Fulop G, Strain JJ, Fahs MC, Schmeidler J, Snyder S. A prospective study of the impact of psychiatric comorbidity on length of hospital stays of elderly medical-surgical inpatients. Psychosom 1998; 39: 273-80.

2. Andrews G, Sanderson K, Slade T, Issakidis C. Why does the burden of disease persist? Relating the burden of anxiety and depression to effectiveness of treatment. Bull World Health Organ 2000; 78: 446-54.

3. Henningsen P, Zimmerman T, Sattel H. Medically unexplained physical symptoms, anxiety and depression. A meta-analytic review. Psychosom Med 2003; 65: 528-33.

4. Ustun T, Sartorius N. Mental illness in general health care: An international study. Chichester: John Wiley \& Sons on behalf of the World Health Organization, 1995.

5. Agudelo D, Lucumí L, Santamaría Y. Evaluación de la depresión en pacientes hospitalizados por distintas enfermedades médicas en la ciudad de Bucaramanga. Pensamiento Psicológico 2008; 4: 59-83.

6. Prina A, Cosco T, Dening T, Beekman A, Brayne C, Huisman M. The association between depressive symptoms in the community, non-psychiatric hospital admission and hospital outcomes: a systematic review. J Psychosom Res 2015; 78: 25-33.

7. World Health Organisation. The global burden of disease-2004 update. Geneve: World Health Organisation, 2004.

8. Campo A, Suárez M. Detección de trastornos depresivos en pacientes hospitalizados (Utilidad de la Escala de Hamilton para depresión). Rev Col Psiquiatría 1998; 27: 220-2.

9. Arancibia M, Behar R. Alexitimia y depresión: evidencias, controversias e implicancias. Rev Chil Neuro-Psiquiat 2015; 53: 24-34.

10. Baéza C, Bulbena A, Fénétrier C, Gély M. Trastornos psicosomáticos y su relación con alexitimia, ansiedad, depresión y demanda de ayuda psicológica. Psicología y Salud 2011; 21: 227-37.

11. Baiardini I, Abbà $S$, Ballauri M, Vuillermoz G, Braido F. Alexithymia and chronic diseases: thestate of the art. G Ital Med Lav Ergon 2011; 33: A47-52.

12. Behar R, Manzo R, Casanova D. Trastornos de la conducta alimentaria y asertividad. Rev Med Chile 2006; 134: 294-301.

13. Cassem H. Depressive disorders in the medically ill. An overview. Psychosom 1995; 36: 2-10.

14. Campo A. La depresión: problema médico cotidiano. Rev Col Psiquiatría 1999; 2: 7-9.

15. Prina AM, Huisman M, Yeap BB, Hankey GJ, Flicker L, Brayne C, et al. Hospital costs associated with depression in a cohort of older men living in Western Australia. Gen Hosp Psychiatry 2014; 36: 33-7.

16. Vásquez C, Sanz J. Fiabilidad y valores normativos de la versión española del Inventario para la Depresión Beck de 1978 en pacientes con trastornos psicológicos. Clínica y Salud 1999; 10: 59-81.

17. Martínez-Sánchez F. Adaptación española de la escala de Alexitimia de Toronto (TAS-20). Clínica y Salud 1996; 7: 19-32.

18. Muldman F. La escala de asertividad de Spencer A Rathus (RAS): Estructura factorial. Terapia Psicológica 1984; 4: 72-7. 
19. Katon W. Clinical and health services relationships between major depression, depressive symptoms, and general medical illness. Biol Psychiatry 2003; 54: 21626.

20. Beck AT, Ward CH, Mendelson M, Mock J, Erbaugh J. An inventory for measuring depression. Arch Gen Psychiatry 1961; 4: 561-71.

21. Palmer S, Vecchio M, Craig J, Tonelli M, Johnson D, Nicolucci A, et al. Prevalence of depression in chronic kidney disease: systematic review and meta-analysis of observational studies. Kidney Int 2013; 84: 179-91.

22. Paykel E, Priest R. Recognition and management of depression in general practice: consensus statement. $\mathrm{Br}$ Med J 1992; 305: 1198-202.

23. Serrano F. Depresión en enfermedades médicas. Anales 2002; 25: 137-48.

24. Alberdi-Sudupe A, Fernández-Díaz F, Iglesias Gil de Bernabé. Depresión en pacientes hospitalizados en el Servicio de Medicina Interna de un Hospital General. Galicia Clin 2013; 74: 161-74.

25. Abúli P, Comas P, Suñer R, Grau A. Niveles de ansiedad y depresión en enfermos hospitalizados y su relación con la gravedad de la enfermedad. Med Clin Barcelona 2003; 120: 370-5.

26. Marchesi C, Ossola P, Tonna M, de Panfilis C. The TAS-20 more likely measures negative affects rather than alexithymia itself in patients with major depres- sion, panic disorder, eating disorders and substance use disorders. Compr Psychiatry 2014; 55: 972-8.

27. Alonso-Fernández F. La alexitimia y su trascendencia clínica y social. Salud Mental 2011; 34: 481-90.

28. Tolmunen T, Lehto SM, Heliste M, Kurl S, Kauhanen J. Alexithymia is associated with increased cardiovascular mortality in middle-aged Finnish men. Psychosom Med 2010; 7: 187-91.

29. Pasini A, Delle Chiaie R, Seripa S, Ciani N. Alexithymia as related to sex, age, and educational level: results of Toronto Alexithymia Scale in 417 normal subjects. Compr Psychiatry 1992; 33: 42-6.

30. Lane R, Sechrest L, Reidel R. Sociodemographic correlates of alexithymia. Compr Psychiatry 1998; 39: 377-85.

31. Parker J, Taylor G, Bagby R. The alexithymia construct: relationship with sociodemographic variables and intelligence. Compr Psychiatry 1989; 30: 434-41.

32. Honkalampi K, Hintikka J, Tanskanen A, Lehtonen J, Viinamäki H. Depression is strongly associated with alexithymia in the general population. J Psychosom Res 2000; 48: 99-104.

33. Shibata M, Ninomiya T, Jensen MP, Anno K, Yonemoto $\mathrm{K}$, Makino S, et al. Alexithymia is associated with greater risk of chronic pain and negative affect and with lower life satisfaction in a general population: the Hisayama study. PLoS ONE 2014; 9: e90984. doi:10.1371/journal. pone.0090984. 